\title{
Comparing Plasma-FIB and Ga-FIB Preparation of Atom Probe Tomography Samples
}

\author{
$\mathrm{K}_{\text {Fisher }}{ }^{1}$, E Marquis ${ }^{1}$ \\ 1. Department of Materials Science and Engineering, University of Michigan, Ann Arbor, MI
}

The site specific lift-out technique has become commonplace for the fabrication of atom probe tomography (APT) specimens from specific regions of interest such as interfaces and grain boundaries (GBs). This procedure typically utilizes a dual-beam scanning electron microscope (SEM) with a focused ion beam (FIB) of $\mathrm{Ga}^{+}$ions used for milling. While this procedure has been successfully used to prepare samples from a large variety of materials including metals, oxides, and ceramics, some materials are susceptible to significant $\mathrm{Ga}^{+}$ion implantation. Aluminum in particular is particularly susceptible to liquid metal embrittlement [1] due to fast $\mathrm{Ga}$ diffusion and decoration of all grain boundaries and interfaces. This phenomenon has been used to reveal the locations of grain boundaries in APT dataset prepared using Ga-FIB milling [2]. However it might also lead to erroneous chemical information. Recent advances in FIB technologies have resulted in the introduction of the plasma-FIB (P-FIB), which utilizes $\mathrm{Xe}^{+}$as the ion source. Typically touted for their higher milling speed compared to $\mathrm{Ga}^{+}$ions due to their increased mass, $\mathrm{Xe}^{+}$ions may also have the advantage of reduced implantation.

In this study, a P-FIB was used to test the feasibility of preparing atom probe specimens that would otherwise be susceptible to $\mathrm{Ga}^{+}$implantation. $\mathrm{Al}-2 \mathrm{Cu}$ was chosen as one of the test materials based on the known issue of $\mathrm{Ga}^{+}$implantation in $\mathrm{Al}$ alloys. The sample was solution-treated at $540^{\circ} \mathrm{C}$ for 4 hours and rapidly quenched in ice water, and then aged at $250^{\circ} \mathrm{C}$ for 4 hours before being polished. GBs were easily identifiable by the channeling contrast of the secondary electrons in the SEM. One grain boundary was chosen for analysis, and two lift-outs were performed; the first on an FEI Helios P-FIB Dual Beam, and the second on an FEI Helios 650 NanoLab Dual Beam using $\mathrm{Ga}^{+}$. The final atom probe specimens from each lift-out were prepared by a series of annular milling steps on the respective instrument. Specimens were field evaporated in a Cameca LEAP 4000X HR using voltage mode with $18 \%$ pulse fraction, $40 \mathrm{~K}$ base temperature, $200 \mathrm{kHz}$ pulse rate, and a target detection rate of 2 ions/1000 pulses. Volumetric reconstructions and subsequent analyses were performed in IVAS 3.6.6 by Cameca.

Figure 1a shows a reconstruction of a specimen prepared using the P-FIB with the GB decorated with $\mathrm{Cu}$ ions. The brackets indicate where the slice shown in Figure $1 \mathrm{~b}$ came from, and the arrow indicates the location of the 1-dimensional concentration profile shown in Figure 1c. As can be seen in 1b, crystallographic information is preserved when preparing in specimens with the P-FIB, and was used to adjust the reconstruction parameters [3]. Figure 1c shows the concentration profile of all ions observed in the mass spectrum decomposed into their elemental forms. This illustrates that $\mathrm{Cu}$ is clearly enriched at the GB, and that Xe was not found at the GB at levels above background noise. In contrast, specimens prepared using the $\mathrm{Ga}^{+}$-FIB display significant levels of Ga throughout the dataset, especially at interfaces. Figure 2a shows an example reconstruction of a specimen containing a $\mathrm{GB}$ and a $\mathrm{Cu}-\mathrm{rich}$ GB precipitate. While the crystallography is still preserved, as shown in Figure $2 \mathrm{~b}$, Ga can be observed in the reconstruction, especially near the top, as indicated by the black dots in (a). A 1-dimensional concentration profile across the GB, shown in Figure 2c, reveals that $\mathrm{Ga}$ is also accumulated at the GB up to about 1 at\%. Ga accumulation was also observed at the matrix/precipitate interface and within the precipitate up to $\sim 1.3 \%$. Further examples will be presented and implications discussed. 


\section{References:}

[1] Hugo, R., and Hoagland, R., Scripta Materialia, 38, (1998), p. 523.

[2] Tang, F. et al., Acta Materialia, 60, (2012), p. 1038.

[3] Gault, B. et al., Microscopy and Microanalysis, 14, (2008), p. 296.

[4] The authors would like to acknowledge the Rickover Fellowship in Nuclear Engineering sponsored by the Naval Reactors Division of the U.S. Department of Energy in conjunction with Bettis Laboratory, West Mifflin, PA for funding this research. The authors would also like to acknowledge Tom Nuhfer at Carnegie Mellon University for providing access to the P-FIB and Bryan Miller for training and helpful discussions, and the University of Michigan College of Engineering and the Michigan Center for Materials Characterization for use of the other instruments.
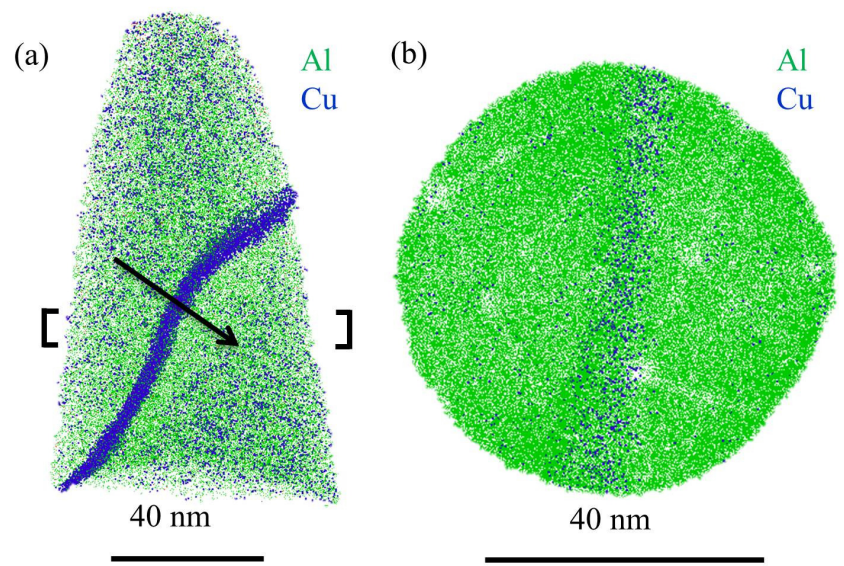

(c)

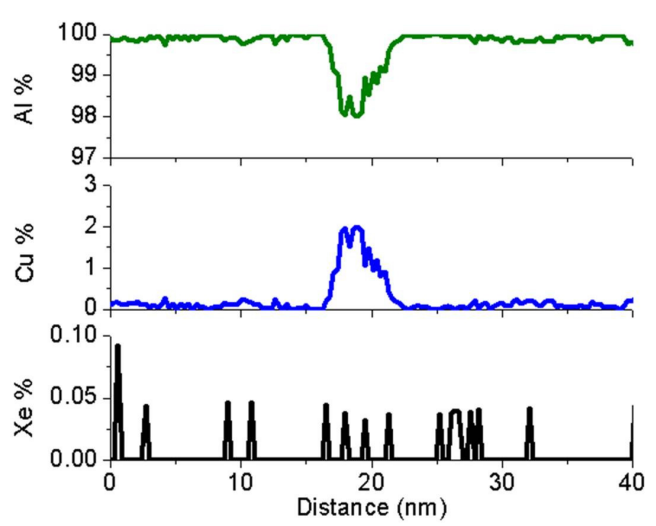

Figure 1. (a) Reconstruction of sample made using P-FIB showing $\mathrm{Al}$ (green) and $\mathrm{Cu}$ (blue) atoms. (b) $10 \mathrm{~nm}$ slice from the bracketed region of (a) showing that cyrstollgraphic data is preserved. (c) 1dimensional concentration profile from the arrowed region of (a) showing $\mathrm{Cu}$ segregation at the GB, and no Xe damage.

(a)

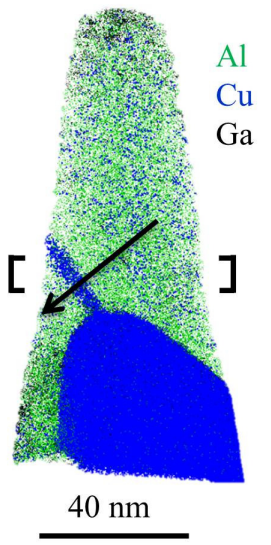

(b)

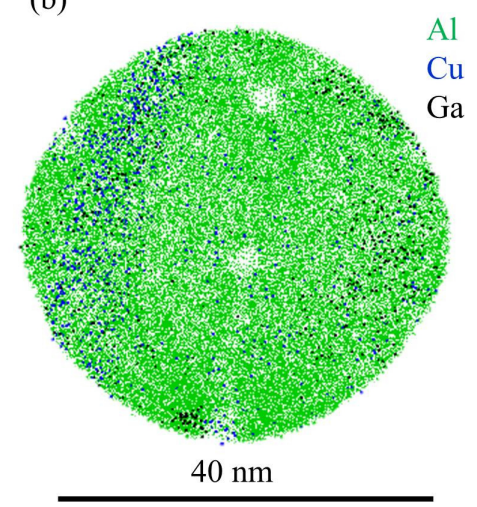

(c)

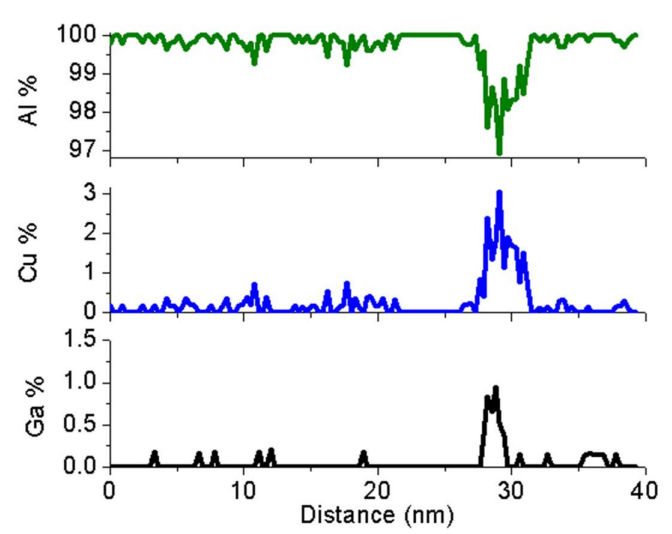

Figure 2. (a) Reconstruction of sample made using $\mathrm{Ga}^{+}-\mathrm{FIB}$ showing $\mathrm{Al}$ (green), $\mathrm{Cu}$ (blue), and $\mathrm{Ga}$ (black) atoms. (b) $10 \mathrm{~nm}$ slice from the bracketed region of (a) with cyrstollgraphic data preserved. (c) 1-dimensional concentration profile from the arrowed region of (a) showing $\mathrm{Cu}$ and $\mathrm{Ga}$ segregation at the GB. 